\title{
VEGF-D expression and lymph vessels play an important role for lymph node metastasis in papillary thyroid carcinoma
}

\author{
Hironao Yasuoka ${ }^{1}$, Yasushi Nakamura ${ }^{1}$, Hui Zuo ${ }^{1}$, Weihua Tang ${ }^{1}$, Yuuki Takamura ${ }^{2}$, \\ Akira Miyauchi ${ }^{2}$, Misa Nakamura ${ }^{1}$, Ichiro Mori ${ }^{1}$ and Kennichi Kakudo ${ }^{1}$ \\ ${ }^{1}$ Department of Pathology, Wakayama Medical University, Wakayama, Japan and ${ }^{2}$ Department of Surgery, \\ Kuma Hospital, Kobe, Japan
}

\begin{abstract}
Papillary thyroid carcinoma frequently metastasizes to regional lymph nodes, and lymph node metastasis increases the risk of local regional relapse. Recent evidence suggests that vascular endothelial growth factor-D (VEGF-D) promotes lymphangiogenesis, which in turn promotes lymphatic metastasis. Therefore, the role of VEGF-D messenger RNA transcript levels and VEGF-D immunoreactivity in lymph node metastasis in papillary thyroid carcinoma was investigated. In addition, the role of blood vascular vessel, lymph vessel, and Flt-4positive vessel densities were studied in relation to their suspected association with lymph node metastasis, and with VEGF-D expression. VEGF-D messenger RNA transcript levels by quantitative real-time reverse transcription-polymerase chain reaction and VEGF-D immunoreactivity by immunohistochemistry in 49 papillary thyroid carcinomas were also studied. This was followed by quantitative immunohistochemical staining for CD34, podoplanin, and Flt-4. Lymph node metastasis was significantly correlated with VEGF-D messenger RNA transcript levels $(P=0.027)$ and VEGF-D immunoreactivity $(P=0.019)$. Increased lymph vessel density was also correlated with VEGF-D expression and lymph node metastasis. In conclusion, our findings indicate that VEGF-D expression and increased lymph vessel density may have an important role for lymph node metastasis in papillary thyroid carcinoma.
\end{abstract}

Modern Pathology (2005) 18, 1127-1133. doi:10.1038/modpathol.3800402; published online 1 April 2005

Keywords: thyroid; papillary carcinoma; VEGF-D; lymph vessel density; metastasis

Papillary thyroid carcinoma metastasizes to regional lymph nodes at a high frequency. ${ }^{1-3}$ In spite of the high incidence of nodal metastasis, the prognostic significance of lymph node metastasis remains controversial. It has also been reported that the presence of nodal metastasis had a significant impact on recurrence only in those patients older than 45 years. ${ }^{4}$ Papillary thyroid carcinoma can metastasize via the blood stream or the lymphatic vasculature, but the mechanisms that determine the route of metastatic spread are largely unknown.

Vascular endothelial growth factor-D (VEGF-D, a novel VEGF member, has been found to induce not only angiogenesis but also lymphangiogenesis via VEGF receptor (VEGFR)-2 and VEGFR-3 (also known as Flt-4 ${ }^{5}$ ). Since VEGFR-3 has been demon-

Correspondence: Dr Y Nakamura, MD, Department of Pathology, Wakayama Medical University, 811-1, Kimiidera, 641-8509 Wakayama City, Japan.

E-mail: ynakamur@wakayama-med.ac.jp

Received 7 January 2005; revised and accepted 20 January 2005; published online 1 April 2005 strated to be expressed almost exclusively in the lymphatic endothelium and thus considered to be a major regulator of lymphangiogenesis, ${ }^{6}$ VEGF-D appears to be an important lymphangiogenic factor. Recent evidence suggests that tumor lymphangiogenesis, that is, the growth of tumor-associated lymphatic vessels, promotes lymphatic metastasis. ${ }^{7}$ Our previous studies have also demonstrated that upregulated VEGF-D expression, and increased Flt4-positive vessel density in human cancer were strongly correlated with lymph node metastasis and unfavorable prognosis. ${ }^{8,9}$

To evaluate the role of VEGF-D in tumor neovascularization and lymph node metastasis in papillary thyroid carcinoma, VEGF-D expression, and blood vessel density, lymph vessel density and Flt-4positive vessel density were assessed in this study. Each microvessel density was studied to assess the growth of tumor-associated vessels. ${ }^{10}$ To evaluate angiogenesis, CD34 expression, which is well known as a specific blood vascular endothelial marker, ${ }^{11,12}$ was used for the determination of blood vessel density. To evaluate lymphangiogenesis, 
podoplanin, a specific lymphatic endothelial marker, ${ }^{13}$ was used for the determination of lymph vessel density. Finally, Flt-4-positive vessel density, which is expressed in lymphatic endothelium of normal tissues and also in blood vascular endothelium of malignant tumor tissues and granulation tissues, ${ }^{6,14}$ was evaluated for VEGF-D-effective vessel density. The relationship between VEGF-D expression and blood vessel density/lymph vessel density/Flt-4-positive vessel density as well as lymph node metastasis was also studied.

\section{Materials and methods}

\section{Patients and Tumor Samples}

The study included 49 patients with papillary thyroid carcinoma, diagnosed and treated in Kuma Hospital, Japan in 2003. All of these cases had no family history of thyroid cancer or malignancy in first-degree relatives as judged by interviews at the time of admission for surgery. The patients had received total or subtotal thyroidectomy with regional lymphadenectomy (central neck dissection, lateral neck dissection, superior mediastinal dissection, or a combination of the above).

All clinical charts and histopathology reports were reviewed for data regarding patient age, sex, tumor size, and presence or absence of lymph node metastasis or distant metastasis. All sections of the papillary thyroid carcinomas and presence or absence of lymph node metastasis were histologically evaluated by three pathologists (HY, YN, and KK). All 49 patients selected for analysis had classical papillary thyroid carcinomas on histopathology. Patients whose tumors showed tall cell or columnar cell differentiation or foci of insular or anaplastic dedifferentiation were not included. Patients with encapsulated papillary thyroid carcinomas or with multifocality were also excluded. Patients and tumor characteristics are shown in Table 1. The median age at surgery for the 49 patients was 48.8 years (range, 16-76 years). In all, $59 \%$ of the patients were older than 45 years $(n=29)$, and $79 \%(n=39)$ of the patients had lymph node metastasis at the time of surgery. A total of 15 cases or $31 \%$ of the patients had N1a lymph node metastasis. There was no correlation between pT and $\mathrm{pN}$. All of the patients have been followed-up and none of the patients has had a recurrence.

\section{Immunohistochemistry}

For immunostainings, paraffin sections of $4-\mu \mathrm{m}$ thickness were deparaffinized. They were then autoclaved for antigen retrieval, and followed by placing in a solution of $97 \%$ methanol and $3 \%$ hydrogen peroxide for $5 \mathrm{~min}$. After washing in PBS, the slides were treated for $20 \mathrm{~min}$ with Protein Block Serum-free (DAKO Co, Carpinteria, CA, USA).
Table 1 Clinico-pathological data for 49 papillary thyroid

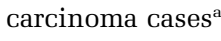

\begin{tabular}{lc}
$\begin{array}{l}\text { Age (years) } \\
<45\end{array}$ & $20(41 \%)$ \\
$\geqslant 45$ & $29(59 \%)$ \\
Sex & \\
Male & $7(14 \%)$ \\
Female & $42(86 \%)$ \\
& \\
Tumor size & \\
pT1 & $7(14 \%)$ \\
PT2 & $32(66 \%)$ \\
pT3 & $4(8 \%)$ \\
PT4 & $6(12 \%)$ \\
& \\
Lymph node metastasis & \\
N0 & $10(21 \%)$ \\
N1a & $15(31 \%)$ \\
N1b & $24(48 \%)$ \\
Distant metastasis & \\
M0 & $49(100 \%)$ \\
M1 & $0(0 \%)$ \\
\hline
\end{tabular}

${ }^{\mathrm{a}}$ Tumor size, Lymph node metastasis, and distant metastasis were classified according to the TNM classification of the UICC, 2002.

Table 2 Antibodies used for immunohistochemistry

\begin{tabular}{lll}
\hline Antibody (supplier) & Antigen & Dilution \\
\hline QBEnd 10 (Dako Co.) & CD34 & $1: 100$ \\
Anti-Podoplanin (AngioBio Co.) & Podoplanin & $1: 100$ \\
Anti-Flt-4 (R\&D Systems, Inc.) & Flt-4 (VEGFR-3) & $1: 50$ \\
Anti-VEGF-D (R\&D Systems, Inc.) & VEGF-D & $1: 100$
\end{tabular}

Primary antibodies were diluted as indicated in Table 2 with $0.3 \%$ BSA in PBS and incubated on the sections at $4^{\circ} \mathrm{C}$. After the overnight treatment with primary antibodies, to avoid the nonspecific biotin reaction, Histofine Simple Stain MAX PO (NICHIREI, Tokyo, Japan) was used as the second antibody for $60 \mathrm{~min}$ according to the manufacturer's instructions. Color was developed using diaminobenzidine with $0.01 \%$ hydrogen peroxide. Hematoxylin was used as a counterstain. For the negative control, all reagents except for the primary antibody were used.

\section{Microscopic Assessment of VEGF-D Expression, Blood Vessel Density, Lymph Vessel Density, and Flt-4-Positive Vessel Density}

The scoring and counts were performed blindly by three investigators who had no clinical knowledge of the patients and prognosis. Evaluation of VEGF-D immunoreactivity was performed according to White et al. ${ }^{15}$ Grading of intensity and extent of staining of the malignant epithelium were as 
follows: $0=$ negative; $1=$ weak/very limited moderate staining; $2=$ moderate widespread/strong localized staining; and $3=$ strong widespread staining. Determination of each microvessel density was performed as described by Weidner et $a l^{10}$ and Dadras et al. ${ }^{16}$ The immunostained sections were scanned by light-microscopy at low magnification $(\times 4)$ and each of the areas of tissue with the greatest number of distinctly highlighted microvessels ('hot spots') were selected. Each microvessel density was then determined by counting all immunostained vessels at a total magnification of $\times 200$ from five areas for each case. The mean number of vessels in each case was evaluated. Since previous reports had suggested that different numbers of lymph vessels were present in intratumoral or peritumoral lesions in human malignant tumors, ${ }^{17-19}$ a zonal analysis of the number of blood vessel density, lymph vessel density, or Flt-4-positive vessel density in intratumoral and peritumoral tissues was performed. Peritumoral microvessels were defined within an area of $100 \mu \mathrm{m}$ from the tumor border. Intratumoral microvessels were defined within the tumor mass and not confined by invagination of normal tissue.

\section{RNA Extraction and Reverse Transcription Reaction}

Messenger RNA (mRNA) was extracted from fresh frozen tumor tissue samples using a QuickPrep micro mRNA purification kit (Amersham Biosciences, Buckinghamshire, UK) according to the protocol provided by the manufacturer. mRNA was reverse-transcribed for single-strand cDNA using Oligo-(dT) $)_{20}$ primer and Thermoscript (Invitrogen, Tokyo, Japan). The reverse transcription (RT) reaction was performed at $55^{\circ} \mathrm{C}$ for $60 \mathrm{~min}$, followed by heating at $85^{\circ} \mathrm{C}$ for $5 \mathrm{~min}$. That only carcinoma tissue was included in freshly frozen thyroid tissue samples was ascertained by studying cryostat sections.

\section{Quantitative Analysis of VEGF-D mRNA by Real-Time Polymerase Chain Reaction}

Transcriptional levels of VEGF-D were measured by quantitative real-time polymerase chain reaction (PCR), similar to the method of Goydos and Gorski, ${ }^{20}$ with some modifications. Assays were performed using universal TaqMan PCR reagents, and the reactions were recorded and analyzed using an ABI Prism 7000 sequence detector equipped with a 96-well thermal cycler (Perkin-Elmer Applied Biosystems, Foster City, CA, USA). ${ }^{21}$ To analyze the ratio of gene transcript levels, we monitored the glyceraldehyde 3-phosphate dehydrogenase (GAPDH) transcripts as an internal control. The ratio of gene transcript levels of each sample $(\Delta C t)$ was evaluated on the basis of its GAPDH transcript content. All experiments were performed in triplicate, and the mean values were calculated (mean
$\Delta C t)$. Inversed $(\Delta \Delta C t)$ values were then used for statistical testing. The cDNA templates were subjected to a 5-min initial denaturation at $95^{\circ} \mathrm{C}$ prior to 40 cycles of PCR $\left(95^{\circ} \mathrm{C}\right.$ for $15 \mathrm{~s}$ and $60^{\circ} \mathrm{C}$ for $1 \mathrm{~min}$, per cycle). The primer and probe mixture for VEGFD or GAPDH were purchased from Perkin-Elmer Applied Biosystems, and PCR was carried out according to the manufacturer's protocol.

\section{Statistical Analysis}

Mann-Whitney $U$-test was used to examine the association of lymph node metastasis with VEGF-D mRNA levels as well as each vessel density. Correlation of VEGF-D immunoreactivity with VEGF-D mRNA levels as well as each vessel density was also investigated by the Mann-Whitney $U$-test. Fisher's exact test was used to examine the association between lymph node metastasis and VEGF-D immunoreactivity. A $P$-value less than 0.05 was considered to be significant. A computer program package (StatView 5.0, Abacus Concepts, Barkeley, CA, USA) was used for all statistical testing and management of the database.

\section{Results}

\section{Immunohistochemistry}

Vessels positive for the blood vascular endothelial marker CD34 were observed in all papillary thyroid carcinoma (Figure 1a and c). They were identified in the endothelium of not only peritumoral vessels but also intratumoral vessels. Mean intratumoral blood vessel density was 170.6/each case, and mean peritumoral vessel density was 114.7/each case. Podoplanin-positive lymph vessels were present in all papillary thyroid carcinoma. All of the stained vessels were typically thin-walled and devoid of red blood cells. Consistent with previous reports, ${ }^{22}$ most lymph vessels were detected in carcinomas with an invasive margin (Figure 1c), and a few of these vessels were identified within the body of the carcinomas (data not shown). Mean intratumoral lymph vessel density was 4.6/each case, and mean peritumoral lymph vessel density was 13.6/each case. Flt-4-positive vessels were present in all papillary thyroid carcinoma. They were identified in the endothelium of not only peritumoral vessels but also intratumoral vessels (Figure $1 \mathrm{~b}$ and e). In peritumoral lesions, most of the Flt-4 expression was observed in the endothelium of podoplanin positive lymphatic vessels. On the other hand, in the intratumoral lesions most of the Flt-4 expression was identified in the endothelium of CD34-positive blood vessels. Mean intratumoral Flt4-positive vessel density was 73.8/each case, and mean peritumoral Flt-4-positive vessel density was 25.9/each case. In papillary thyroid carcinoma cells, the cytoplasmic expression of VEGF-D protein was 

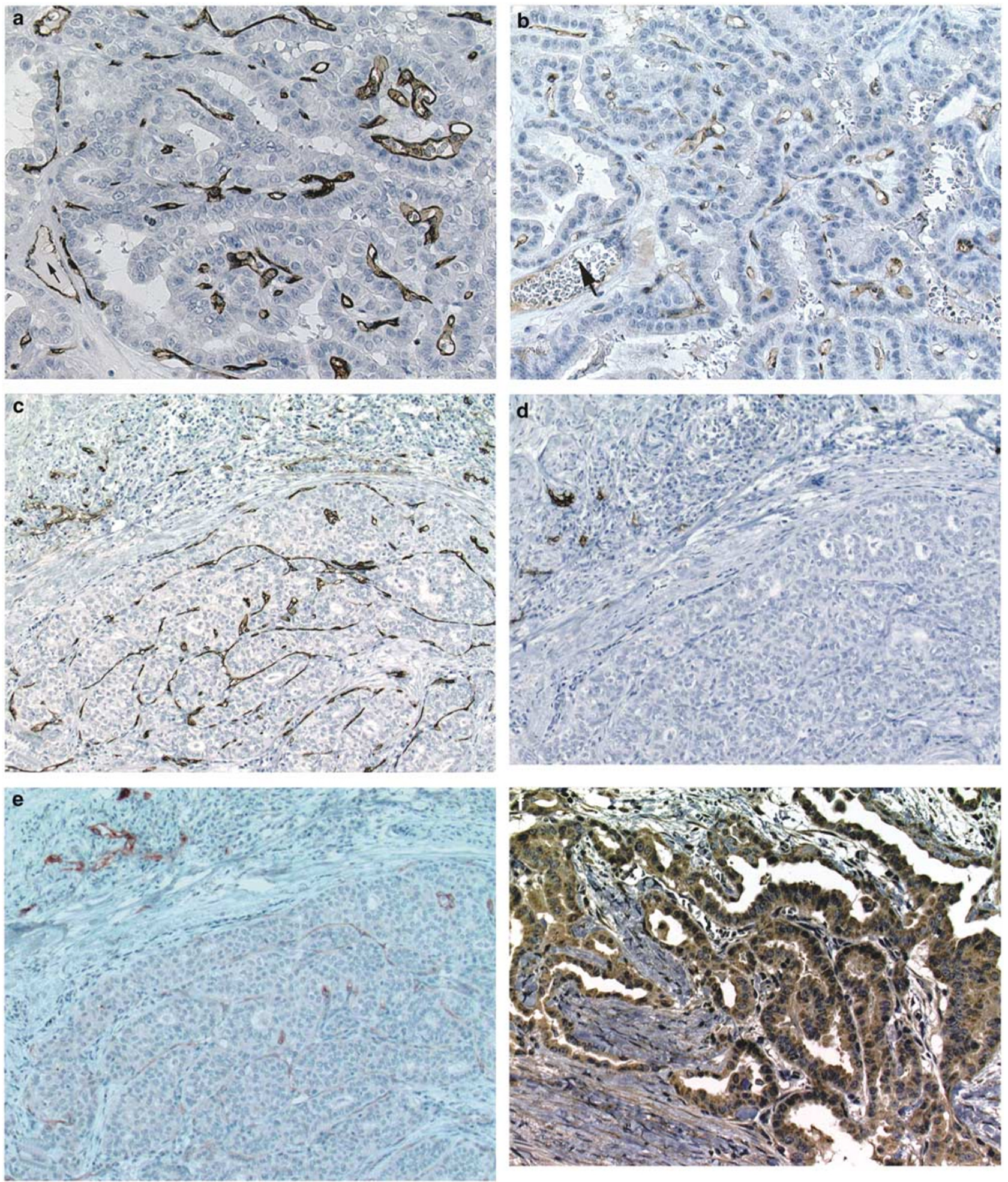

Figure 1 Immunohistochemical staining of consecutive sections in intratumoral lesions of papillary thyroid carcinoma for CD34 (a) and Flt-4 (b). In intratumoral lesions, most of the Flt-4 expression was observed in the endothelium of CD34-positive blood vessels (arrows; vein, $\times 200$ ). Immunohistochemical staining of consecutive sections in peritumoral lesions of papillary thyroid carcinoma for CD34 (c), Podoplanin (d), and Flt-4 (e). Most podoplanin-positive vessels were detected in carcinomas with an invasive margin. In peritumoral lesions, Flt-4 expression was observed not only in intratumoral lesions but also in peritumoral lesions $(\times 100)$. VEGF-D expression patterns in papillary thyroid carcinoma (f). Almost all tumor cells showed diffuse and intense immunostaining $(\times 200)$. 
observed not only in the malignant (Figure 1f) but also in the normal thyroid follicular cells (data not shown). The staining noted in the normal follicular epithelial cells was always of low grade (Grades $0-1$ ), whereas in the tumor cells, the staining was both low and high grade (Grades 1-3). For statistical analysis, VEGF-D antibody-stained sections were grouped as low grade (0-2) and high grade. ${ }^{3}$ According to this criteria, high-grade VEGF-D expression was observed in $37 \%(18 / 49)$ of the 49 papillary thyroid carcinomas.

\section{Statistics}

The results are summarized in Tables 3 and 4 . As shown in Table 3 , a significant correlation was found between VEGF-D mRNA transcript levels or immunoreactivity and lymph node metastasis $(P=0.027$ and 0.019 , respectively), and VEGF-D immunoreactivity tended to be correlated with
VEGF-D transcript levels $(P=0.059)$. VEGF-D immunoreactivity was significantly and positively associated with both intratumoral and peritumoral lymph vessel densities $(P=0.011$ and 0.046 , respectively). No significant correlations were detected between VEGF-D immunoreactivity and blood vessel density (both intratumoral and peritumoral) or Flt-4-positive vessel density (both intratumoral and peritumoral). As shown in Table 4, both intratumoral and peritumoral lymph vessel densities were significantly associated with lymph node metastasis $(P=0.028$ and 0.001 , respectively). No significant correlations emerged between lymph node metastasis and blood or Flt-4-positive vessel density.

\section{Discussion}

Tumor metastasis may depend on the capacity of tumor cells to induce angiogenesis and/or lymphangiogenesis. VEGF-D, a potent angiogenic factor

Table 3 Relationship between VEGF-D expression and lymph node metastasis or vessel density ${ }^{\mathrm{a}}$

\begin{tabular}{|c|c|c|c|}
\hline \multirow[t]{2}{*}{ Factors } & \multicolumn{2}{|c|}{ Lymph node metastasis } & \multirow[b]{2}{*}{ P-value } \\
\hline & \multicolumn{2}{|c|}{ (Mean levels/ \pm s.d.) } & \\
\hline VEGF-D mRNA transcript levels & $0.078 \pm 0.034$ & $0.082 \pm 0.043$ & 0.027 \\
\hline $\begin{array}{l}\text { VEGF-D (-) } \\
\text { Immunoreactivity (+) }\end{array}$ & $\begin{array}{l}10 \text { cases } \\
0 \text { cases }\end{array}$ & $\begin{array}{l}21 \text { cases } \\
18 \text { cases }\end{array}$ & 0.019 \\
\hline \multirow[t]{2}{*}{ Factors } & \multicolumn{2}{|c|}{ VEGF-D immunoreactivity } & \multirow[t]{2}{*}{ P-value } \\
\hline & \multicolumn{2}{|c|}{ (Mean vessel density/field \pm s.d.) } & \\
\hline Intratumoral blood vessel density & $169.1 \pm 30.3$ & $173.1 \pm 45.4$ & 0.671 \\
\hline Peritumoral blood vessel density & $113.5 \pm 32.4$ & $116.9 \pm 35.3$ & 0.772 \\
\hline Intratumoral lymph vessel density & $3.5 \pm 6.4$ & $6.5 \pm 5.8$ & 0.011 \\
\hline Peritumoral lymph vessel density & $12.0 \pm 9.1$ & $16.4 \pm 7.6$ & 0.046 \\
\hline Intratumoral Flt-4-positive vessel density & $72.0 \pm 36.3$ & $77.1 \pm 33.7$ & 0.604 \\
\hline Peritumoral Flt-4-positive vessel density & $24.1 \pm 19.9$ & $28.9 \pm 18.6$ & 0.340 \\
\hline
\end{tabular}

${ }^{a}$ Mann-Whitney $U$-test was used to examine the association of lymph node metastasis with VEGF-D mRNA levels, or the association of VEGF-D immunoreactivity with each vessel density. Fisher's exact test was used to examine the association between lymph node metastasis and VEGF-D immunoreactivity.

Table 4 Relationship between lymph node metastasis and vessel density ${ }^{a}$

\begin{tabular}{|c|c|c|c|}
\hline \multirow[t]{2}{*}{ Factors } & \multicolumn{2}{|c|}{ Lymph node metastasis } & \multirow[t]{2}{*}{$\mathrm{P}$-value } \\
\hline & \multicolumn{2}{|c|}{ (Mean vessel density/field \pm s.d.) } & \\
\hline Intratumoral blood vessel density & $170.9 \pm 30.2$ & $170.5 \pm 38.0$ & 0.951 \\
\hline Peritumoral blood vessel density & $109.0 \pm 28.3$ & $116.2 \pm 34.5$ & 0.629 \\
\hline Intratumoral lymph vessel density & $1.3 \pm 2.3$ & $5.4 \pm 6.8$ & 0.028 \\
\hline Peritumoral lymph vessel density & $5.3 \pm 3.7$ & $15.8 \pm 8.4$ & $<0.001$ \\
\hline Intratumoral Flt-4-positive vessel density & $79.8 \pm 36.5$ & $72.3 \pm 35.1$ & 0.577 \\
\hline Peritumoral Flt-4-positive vessel density & $21.9 \pm 13.6$ & $26.9 \pm 20.6$ & 0.719 \\
\hline
\end{tabular}

${ }^{\mathrm{a}}$ Mann-Whitney $U$-test was used to examine the association of lymph node metastasis with each vessel density. 
in vivo, which stimulates endothelial cell proliferation and migration, ${ }^{5}$ is involved in promoting tumor angiogenesis and lymphangiogenesis. ${ }^{7}$ VEGF-D is also known to be required for the growth and establishment of lymphatic vessels within tumors. However, the absence of specific markers for lymphatic vessels has made their identification difficult. Recently, podoplanin, an $\sim 38 \mathrm{kDa}$ membrane mucoprotein originally detected on the surface of rat podocytes, ${ }^{13}$ was established as a specific marker for lymphatic endothelium. ${ }^{23}$ This discovery changed the landscape for lymphatic studies, with podoplanin being used as a valuable marker for identifying lymph vessels and accurately evaluating lymph vessel density. ${ }^{17,18,24-26}$

In this study, we found that lymph node metastasis was statistically correlated with both increased lymph vessel density and VEGF-D expression of both mRNA and protein, and that VEGF-D immunoreactivity was significantly and positively correlated with increased lymph vessel density. Our study is the first to demonstrate that there was a significant correlation between VEGF-D expression and lymph vessel density, or between VEGF-D expression and lymph node metastasis, in human papillary thyroid carcinoma. These results concur with previous animal studies that VEGF-D plays an important role in lymph node metastasis via lymphangiogenesis. ${ }^{7}$ An earlier report using human papillary thyroid carcinoma also supported our present observation that the presence of lymphatics in papillary thyroid carcinoma was significantly associated with nodal metastasis. ${ }^{22}$

VEGF-D expression is upregulated in a number of other types of tumors. ${ }^{8,15}$ In this study, we found that increased VEGF-D expression was correlated with lymph node metastasis in papillary thyroid carcinoma. Tanaka et al, ${ }^{27}$ however, were unable to find a significant relationship between VEGF-D expression and lymph node metastasis. As mentioned earlier, VEGF-D immunoreactivity can also be detected in normal thyroid follicular epithelium. We carefully confirmed that only carcinoma tissue was included in the freshly frozen thyroid tissue samples by viewing cryostat sections. Therefore, the RT-PCR assay used by Tanaka et al may not have revealed a true and specific expression of VEGF-D in tumor cells if a carefully conducted laser microdissection was not utilized to obtain only tumor cells for their analyses. Furthermore, Tanaka et al found VEGF-D expression in only about $52 \%$ of the tumor tissues investigated using a semiquantitative RT-PCR method. This consisted of electrophoresis in agarose gel, scanning the bands of the positive films and measuring the density and the width of each of the PCR products. As a result of the higher sensitivity of using real-time PCR to detect PCR transcripts, VEGF-D mRNA expression was detected in all papillary thyroid carcinomas in our study.

In conclusion, VEGF-D expression was correlated with increased lymph vessel density, and may have an important role for lymphangiogensis in papillary thyroid carcinoma. VEGF-D expression and increased lymph vessel density was also associated with lymph node metastasis, and may play an important role in the progression of lymph node metastasis.

\section{Acknowledgements}

We thank Mr Hiroshi Yoshida, Kuma Hospital, for his help in preparing tissue samples for this study, and Mrs Emiko Taniguchi, Department of Pathology, Wakayama Medical University, for expert technical assistance.

\section{References}

1 Shaha AR, Shah JP, Loree TR. Patterns of nodal and distant metastasis based on histologic varieties in differentiated carcinoma of the thyroid. Am J Surg 1996;172:692-694.

2 Kakudo K, Tang W, Ito Y, et al. Parathyroid invasion, nodal recurrence, and lung metastasis by papillary carcinoma of the thyroid. J Clin Pathol 2004;57: 245-249.

3 Kakudo K, Tang W, Ito Y, et al. Papillary carcinoma of the thyroid in Japan: subclassification of common type and identification of low risk group. J Clin Pathol 2004;57:1041-1046.

4 Hughes CJ, Shaha AR, Shah JP, et al. Impact of lymph node metastasis in differentiated carcinoma of the thyroid: a matched-pair analysis. Head Neck 1996;18: 127-132.

5 Achen MG, Jeltsch M, Kukk E, et al. Vascular endothelial growth factor D (VEGF-D) is a ligand for the tyrosine kinases VEGF receptor 2 (Flk1) and VEGF receptor 3 (Flt4). Proc Natl Acad Sci USA 1998;95: 548-553.

6 Kaipainen A, Korhonen J, Mustonen T, et al. Expression of the fms-like tyrosine kinase 4 gene becomes restricted to lymphatic endothelium during development. Proc Natl Acad Sci USA 1995;92: 3566-3570.

7 Stacker SA, Caesar C, Baldwin ME, et al. VEGF-D promotes the metastatic spread of tumor cells via the lymphatics. Nat Med 2001;7:186-191.

8 Nakamura Y, Yasuoka H, Tsujimoto M, et al. Prognostic significance of vascular endothelial growth factor D in breast carcinoma with long-term follow-up. Clin Cancer Res 2003;9:716-721.

9 Nakamura Y, Yasuoka H, Tsujimoto M, et al. Flt-4positive vessel density correlates with vascular endothelial growth factor-D expression, nodal status, and prognosis in breast cancer. Clin Cancer Res 2003;9:5313-5317.

10 Weidner N, Semple JP, Welch WR, et al. Tumor angiogenesis and metastasis-correlation in invasive breast carcinoma. N Engl J Med 1991;324:1-8.

11 Appleton MA, Attanoos RL, Jasani B. Thrombomodulin as a marker of vascular and lymphatic tumours. Histopathology 1996;29:153-157.

12 Miettinen M, Lindenmayer AE, Chaubal A. Endothelial cell markers CD31, CD34, and BNH9 antibody to 
$\mathrm{H}$ - and Y-antigens-evaluation of their specificity and sensitivity in the diagnosis of vascular tumors and comparison with von Willebrand factor. Mod Pathol 1994;7:82-90.

13 Breiteneder-Geleff S, Matsui K, Soleiman A, et al. Podoplanin, novel 43-kd membrane protein of glomerular epithelial cells, is down-regulated in puromycin nephrosis. Am J Pathol 1997;151:1141-1152.

14 Partanen TA, Arola J, Saaristo A, et al. VEGF-C and VEGF-D expression in neuroendocrine cells and their receptor, VEGFR-3, in fenestrated blood vessels in human tissues. FASEB J 2000;14:2087-2096.

15 White JD, Hewett PW, Kosuge D, et al. Vascular endothelial growth factor-D expression is an independent prognostic marker for survival in colorectal carcinoma. Cancer Res 2002;62:1669-1675.

16 Dadras SS, Paul T, Bertoncini J, et al. Tumor lymphangiogenesis: a novel prognostic indicator for cutaneous melanoma metastasis and survival. Am J Pathol 2003;162:1951-1960.

17 Nakamura Y, Yasuoka H, Tsujimoto M, et al. Lymph vessel density correlates with nodal status, VEGF-C expression, and prognosis in breast cancer. Breast Cancer Res TR (in press).

18 Birner P, Schindl M, Obermair A, et al. Lymphatic microvessel density as a novel prognostic factor in early-stage invasive cervical cancer. Int J Cancer 2001;95:29-33.

19 Straume O, Jackson DG, Akslen LA. Independent prognostic impact of lymphatic vessel density and presence of low-grade lymphangiogenesis in cutaneous melanoma. Clin Cancer Res 2003;9:250-256.
20 Goydos JS, Gorski DH. Vascular endothelial growth factor C mRNA expression correlates with stage of progression in patients with melanoma. Clin Cancer Res 2003;9:5962-5967.

21 Heid CA, Stevens J, Livak KJ, et al. Real time quantitative PCR. Genome Res 1996;6:986-994.

22 Hall FT, Freeman JL, Asa SL, et al. Intratumoral lymphatics and lymph node metastases in papillary thyroid carcinoma. Arch Otolaryngol Head Neck Surg 2003;129:716-719.

23 Breiteneder-Geleff S, Soleiman A, Kowalski H, et al. Angiosarcomas express mixed endothelial phenotypes of blood and lymphatic capillaries: podoplanin as a specific marker for lymphatic endothelium. Am J Pathol 1999;154:385-394.

24 Birner P, Obermair A, Schindl M, et al. Selective immunohistochemical staining of blood and lymphatic vessels reveals independent prognostic influence of blood and lymphatic vessel invasion in early-stage cervical cancer. Clin Cancer Res 2001;7:93-97.

25 Birner P, Schindl M, Obermair A, et al. Lymphatic microvessel density in epithelial ovarian cancer: its impact on prognosis. Anticancer Res 2000;20: 2981-2985.

26 Schoppmann SF, Birner P, Studer P, et al. Lymphatic microvessel density and lymphovascular invasion assessed by anti-podoplanin immunostaining in human breast cancer. Anticancer Res 2001;21:2351-2355.

27 Tanaka K, Kurebayashi J, Sonoo H, et al. Expression of vascular endothelial growth factor family messenger RNA in diseased thyroid tissues. Surg Today 2002;32: 761-768. 